\title{
Analysis to Determine the Scope and Challenging Responsibilities of Ethical Hacking Employed in Cyber Security
}

\author{
R. Vignesh ${ }^{1 *}$, K. Rohini ${ }^{2}$ \\ ${ }^{1}$ Student, Electronic and Communication Engineering, SASTRA, Tanjore, India. \\ ${ }^{2}$ Assistant Professor, Department of Information Technology, School of Computing Sciences, VISTAS, Chennai, India. \\ E-mail:rrohini16@gmail.com \\ *Corresponding author E-mail:rrvignesh10@ gmail.com
}

\begin{abstract}
This paper analyzes a variety of Challenging Roles of Ethical Hacking employed in Cyber Security. The requirement for more viable requesting data security rehearses is progressively evident with every security encroaches revealed in the media. Ethical hacking set forward a target investigation of an association's data security bearing for associations of numerous phase of security capability. Programmers must output for shortcomings, test section focuses, needs targets, and build up a procedure that best use their assets. The reason for this sort of security appraisal directly affects the estimation of the entire assessment. More finished it is recognized that electronic devices are fundamental to forestall digital culprits hacking into online systems to contain their administrations and access secret information for uncalled for purposes. Ethical Hacking is capably required where approved programmers endeavor to penetrate a business' frameworks/arranges for the benefit of the proprietors with the goal of discovering security shortcomings. It give bits of knowledge into how Ethical Hacking, as Penetration Testing utilizing free open source devices, can be utilized by associations to secure their system's administrations/activities. Utilizing Nmap, Google Hacking, Nessus, Brutus and Acunetix .Thus measures were placed in to determine these vulnerabilities and dodge the delicate information from potential digital threats.
\end{abstract}

Keywords: Ethical hacking, cyber security, penetration testing, vulnerabilities, foot printing, scanning.

\section{Introduction}

The term Ethical hacking refers to the process of finding the weaknesses and vulnerabilities of computer and information systems by duplicating the intent and actions of malicious hackers. The term Ethical hacking alludes to the way toward finding the shortcomings and vulnerabilities of PC and data frameworks by copying the aim and activities of malignant programmers. It includes utilizing PC hacking abilities to distinguish organize security vulnerabilities and fix security openings before anybody can manhandle them. It is otherwise called entrance testing, interruption testing, or red joining. When all is said in done hacking is of two sorts in particular malevolent hacking, ethical hacking or now and again known as white cap and dark cap hacking separately [1].

An ethical programmer is a PC and systems administration master who takes after ethical standards and deliberately endeavours to enter a PC framework or system for its proprietors to find security vulnerabilities that a noxious programmer could conceivably misuse. For hacking to be regarded ethical, the programmer must have the consent from the proprietor of the association to test their system and endeavour to distinguish potential security dangers. As of late, numerous associations have confronted digital assaults prompting the developing need of having proficient ethical programmers who can shield their systems.

\section{Security Issues}

\section{Security}

Not a capacity, however everyone's activity. Security ought to be taught in each training and capacity of the IT office. For e.g. engineers ought to comprehend the significance of secure coding. This aids in overseeing security comfortable root at the coding level. Understanding the distinctive manners by which the code can be traded off and after that circling security, can be a best practice. The system administrators can investigate secure system models. Each capacity in IT should think from a security edge to whatever they purchase, do and execute. To the extent sprouting security experts are concerned, building up a profound specialized area aptitude is a must.[2]

\section{a. Security issues in networking}

The biggest issue in networking is security problems. The hackers hijack the individuals, organizations and countries information. In the Cyber Warfare the Nation States are penetrating other nation's computers [3].

\section{b. Security issues in cloud computing}

In the field of Cloud computing, many new cloud security issues prevails among the Application service providers(ASPs), Cloud 
Service Providers(CSPs), Internet Service Providers(ISPs) and Users trust domains. Cloud Computing and internet such as Applications through internet (Google Docs)

Computing through internet (Amazon EC3) must be secured.

\section{c. Issues with the storage and backup through internet}

Security violation is in every part of data transactions. Even the Mobile devices (Android) got security violated and attacked by Trojans. Policy, Security, and Quality of Service issues has to be resolved in a Multi-organizational ownership. The security issue can be resolved by the Separation of Control and Data Planes [4].

\section{d. Venture security}

Venture Security is mind boggling and consistently advancing. The cost of security should not exclusively be thought about remembering the break situation yet additionally the advanced guide of the association. This sheer yearning of organizations to interface with their clients, representatives, investors and different partners, through advanced means carries alongside it, a natural danger of security as well. The interests in these advanced arrangements additionally bring the cost of security under its domain.

As a characteristic movement, the inquiry emerges on the method of reasoning for putting resources into security when once a day, there are no real security episodes included. It's an indistinguishable reason from to why security like safety belt and protective cap exists. So also, there are a plenty of security techniques in different businesses like carriers, fabricating and so on [5].

The primary concern is data security turns into a piece of the business when it is contemplated all the while with the advanced way the organization has envisioned. Henceforth, security never again remains an idea in retrospect.

\section{Challenges for Cisos}

In the wake of expanding digitalization, accessibility of the required IT security spending plan isn't a test for CISOs. The test is the absence of comprehension in choosing why a specific security apparatus is valuable. Numerous security experts do not have the expertise of perceiving, understanding the issue and afterward having the correct perspective to discover an answer. Despite the fact that a firewall is as of now introduced an Intrusion Prevention System (IPS) is basically required for certain profound bundle assaults on the system. The security experts ought to have an inside and out comprehension of the security items and necessities to see how the assaults are completed, distinguished, caught and after that remediated. In any case, tragically, this comprehension is absent in a few associations without the entrance testing which can get to the foundation of the issue. The issues are comprehended completely and a near idiot proof arrangement design is proposed.

The improvement is in the fallout of the current spate of payment product assaults and furthermore because of the forcing dangers that wait as cybercrime has turned out to be more sorted out.

\section{Capability of AI in Comprehending Security Issues}

$\mathrm{AI}$ and ML have colossal extension in explaining the data security challenges and there are merchants who are as of now guaranteeing ML abilities in their answers. Indeed, even programmers are endeavouring to utilize AI to organize assaults. The respondents for this situation, should do get up to speed. AI has gigantic potential however catching the information from different hubs is basic. Information can either be caught, prepared from one point or at different hubs. The Endpoint conduct examination instruments exists and profound apparatuses, with cutting edge investigation abilities that can procedure information midway as well. AI will have an edge over different instruments in isolating the false positives from the suspicious activity.

\section{Security Tools}

Wireshark (packet sniffer previously known as Ethereal)

Metasploit (exploit)

Nessus (vulnerability scanner)

Aircrack (WEP and WPA wafer)

Grunt (organize interruption indicator)

Cain and Abel (parcel sniffer and secret key wafer)

Netcat (debugger and investigation device)

tcpdump (parcel sniffer)

John The Ripper (parcel sniffer and secret key saltine)

Acunetix (web defencelessness scanner WVS) that sweeps and discovers the defects in a site that could turn out to be lethal.

Nmap. (System Mapper) is a free and open source (permit) utility for arrange revelation and security examining.

The penetration testing has been performed by the ethical hacking people to know the vulnerability and exploit them. [6].

\section{Penetration Testing}

The entrance testing has turned out to be progressively main stream by both cybercriminals (to get to secret data) and ethical programmers (to find and survey the shortcomings in an organization's own systems) [7] [8].

Deciding Assessment Scope:- The principle objective of the infiltration (pen) test before beginning any ethical hacking work is to decide the extent of the task. Extension crawl is the development of the task past its unique particular. The customer should need to grow the pen test past its unique particulars.

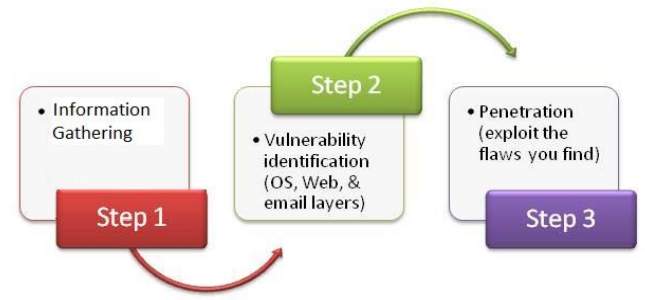

Fig. 1: Proposed procedure of ethical hacking

\section{Foot Printing and Scanning-Information Gathering}

At first the objective is precisely broke down by the Ethical hacking process. The association, its qualities and shortcomings, its responsiveness to the unforeseen, and all the data expected to decide and build up the assault are gathered. The data is accumulated specifically from the organization itself and additionally from open sources. At that point the checking procedure begins with the site or framework for vulnerabilities and shortcomings they can later adventure for the focused on assault.

Foot printing is about data assembling and is both inactive and dynamic. Checking on the organization's site is a case of uninvolved foot printing, while calling the assistance work area and endeavouring to social building them out of favoured data is a case of dynamic data gathering. Filtering involves pinging machines, deciding system ranges and port checking singular frameworks.

The data gathering ventures of foot printing and checking are of most extreme significance. Great data social affair can have the effect between a fruitful pen test and one that has neglected to give most extreme advantage to the customer. An astonishing measure of data is accessible about most associations in business today. This data can be found on the association's site, exchange papers, 
Usenet, money related databases, or even from displeased workers.

\section{Enumeration}

Improvement of the methodology for the assault has been finished. With the data accumulated up until now, they can choose which instruments and strategies to use to best hit the framework. Social designing assaults, SQL infusions, malware must be looked over a wide range.

\section{Penetration}

The last stage is simply the assault. Utilizing the devices or methods distinguished in the past advance, ethical programmers abuse the weakness to break into the association.

Once the test is finished, ethical programmers as a rule furnish the association with a nifty gritty report of the vulnerabilities found, the portrayal of the assault they performed and prescribed activities to make the association more secure.[9].

\section{Findings and Discussion}

In spite of the fact that there are well ordered technique for data assembling the procedure of ethical hacking is extremely a procedure of disclosure. Kali Linux is always developing with new highlights being added to the appropriation constantly, which is one of best Penetration Testing Tools.

Infiltration Testing is a strategy for testing in which the territories of shortcoming are found in the product frameworks as far as security, which are Performed for Websites or Servers or Networks by ethical programmers or Security Testers.

- It begins with a rundown of Vulnerabilities or potential issue zones that would cause a security break for the framework.

- If conceivable, this rundown of things is positioned in the request of need/criticality.

- Devise infiltration tests that would work (assault your framework) from both inside the system and outside (remotely) are done to decide whether you can get to information/arrange/server/site unapproved.

- If unapproved get to is conceivable, at that point the framework must be amended and the arrangement of steps should be re-keep running until the point when the issue region is settled.

The pen-testing isn't the same as powerlessness testing. The aim of weakness testing is simply to distinguish the potential issues, though pen-testing is to assault those issues.

Ethical Hacking Methodology of joined footprinting and checking stages is in Penetration Testing. A definitive point of this stage was to pick up information identifying with the objective web have utilizing Nmap[9].

Nmap was used to make a network survey to assemble knowledge with reference to hosts, ports, host operational systems and network topologies. [14][15]

Initially, all of the hosts on the network were known, as well as all the devices connected to the network (PC's, servers and routers).

Further analysis of every network host proved the necessity for extra data with reference to the online server and therefore the native servers known. So Nmap scans were targeted onto these 2 hosts to assemble further knowledge as well as port numbers, protocols and therefore the services they were running. By getting this extra knowledge it raised the chance of network weaknesses being detected throughout the following part of this penetration check [10][11].

Nmap ("Network Mapper") could be a free and open supply (license) utility for network discovery and security auditing. several systems and network directors conjointly notice it helpful for tasks love network inventory, managing service upgrade schedules, and watching host or service time period. Nmap uses raw IP packets in novel ways in which to work out what hosts area unit accessible on the network, what services (application name and version) those hosts area unit giving, what operational systems (and OS versions) they're running, what sort of packet filters/firewalls area unit in use, and dozens of alternative characteristics. it had been designed to quickly scan giant networks, however works fine against single hosts. Nmap runs on all major laptop operational systems, and official binary packages area unit accessible for UNIX, Windows, and macintosh OS X. additionally to the classic command-line Nmap practicable, the Nmap suite includes a sophisticated GUI and results viewer (Zenmap), a versatile knowledge transfer, redirection, and debugging tool (Ncat), a utility for scrutiny scan results (Ndiff), and a packet generation and response analysis tool (Nping). Commands utilized in for Bypass Firewalls victimisation nmap area unit as below:

nmap -sn -v -pn victim's IP/Host name/website

nmap -sn - $v$-pn hostname.com [12][13]

the information gathered from Nmap was utilised throughout the vulnerability detection part aimed toward increasing the irresponsibleness of the penetration check. Additionally, by aggregation knowledge from the target systems, it provided insight into however the network operates. Nmap was named "Security Product of the Year" by UNIX Journal, information World and LinuxQuestions.Org.

\section{Conclusion}

The organizations will use penetration testing to find potential security flaws and vulnerabilities to guard their business network. Once known it's doable to place measures in situate to resolve them. Moreover, it had been conjointly discovered that it's helpful to utilize multiple free penetration testing tools to extend the chance of locating the network vulnerabilities and by finding them to stop a malicious hacker from getting the sensitive and confidential knowledge. Penetration tests discovered if any of the known vulnerabilities might are exploited by malicious hackers. this could involve hacking into the host's networks and making an attempt to step up the privileges almost like a black-hat hacker. By victimisation free open supply tools little businesses with restricted resources will manage more practical cyber security. we are able to say that the information collected from the penetration check (e.g. combination of Nmap, Google Hacking, Nessus and Brutus) allowed a corporation to boost their network security facilities and stop unauthorized access by black-hat hackers via cyber attacks. So the utilization of free open supply code are often even to boost the protection of organizations networks to reinforce their cyber security procedures.

\section{References}

[1] Lawson B, FBI Recruiting 'Ethical Hackers' To Investigate Cyber Crime. http://www.newsy.com

[2] Surendar, A. (2018, January 1). Letter from the desk of editor's. International Journal of Pharmaceutical Research, 10(1).

[3] Aggarwal P, Arora P, Neha P \& Poonam S, "Review on Cyber Crime and Security", Int. J. Res in Eng and Allied Scs., Vol.2,No.1, (2014), pp.48-51.

[4] http://www.cse.wustl.edu/ jain/talks/adcons.htm

[5] Oriyano S, Ceh: Certified ethical Hacker Study Guide, John Wiley, Indianapolis, (2014).

[6] Prasad M \& Manjula B, “ethical Hacking Tools: A Situational Awareness", Int J. rising detective. Comp. Sc. \& Elec., Vol.11, (2014), pp.33-38.

[7] Villalobos Antúnez, JV (2017). Karl R. Popper, Heráclito y la invención del logos. Un contexto para la Filosofía de las Ciencias Sociales. Opción Vol. 33, Núm. 84. 5-11

[8] Chow E, "ethical Hacking \& Penetration Testing”, ACC 626: IT analysis Paper, Vol.1, No.1, (2011). 
[9] Engebretson $\mathrm{P}$, the fundamentals of Hacking and Penetration Testing, Elsevier, USA, (2013)

[10] Kirsch C, Introduction to Penetration Testing.

[11] //.community.rapid7.com

[12] Long J, Gardener B \& Brown J, Google Hacking for Penetration Testers, Syngress commercial enterprise, USA, (2007).

[13] Lyon G, Nmap Network Scanning, Insecure, USA, (2009).

[14] http://www.ankitfadia.in/afceh

[15] http://tools.kali.org/

[16] A Akhmetbekova, P Auyesbayeva, Sh Ibrayev (2018). Turkic "Hikaya" genre and its characters. Opción, Año 33. 81-106. 